\title{
Tratamento das gigantomastias
}

\section{Treatment of gigantomasty}

Fernando Sanfelice André ${ }^{1}$

Ana Carolina Chociai ${ }^{2}$

Trabalho realizado no Serviço de Cirurgia Plástica do Hospital

Regional Hans Dieter Schmidt, Joinville, SC, Brasil

Artigo submetido pelo SGP (Sistema de Gestão de Publicações) da RBCP.

Artigo recebido: 9/10/2010 Artigo aceito: 8/11/2010

\section{RESUMO}

Apresentamos a conduta adotada para o tratamento de 200 pacientes portadoras de gigantomastia. As técnicas e táticas cirúrgicas utilizadas e os resultados obtidos são discutidos.

Descritores: Mama/cirurgia. Mamoplastia. Cirurgia Plástica.

\section{ABSTRACT}

We presented our conduct for the treatment of 200 patients with gigantomasty, our techniques, tactical surgeries and results, for this mammary pathology.

Keywords: Breast/surgery. Mammaplasty. Plastic surgery.

\section{INTRODUÇÃO}

A gigantomastia é uma condição não rara, caracterizada por um aumento excessivo do volume das mamas, que pode provocar danos físicos e psicológicos para as pacientes. Os sintomas incluem mastalgia, ulceração, infecção submamária, problemas posturais, cervicalgia, dorsalgia e injúria por tração crônica dos $4^{\circ}, 5^{\circ}$ e $6^{\circ}$ nervos intercostais, provocando perda da sensibilidade mamária. A gigantomastia está também associada com o déficit de crescimento fetal durante a gestação.

Até este momento, não há classificação ou definição universal aceita para gigantomastias. Muitos autores citam a gigantomastia como um aumento da mama que requer redução de mais de 1500 g por mama. Entretanto, há uma discordância na literatura com o peso do material ressecado. Poucos trabalhos procuraram classificar as mamas a partir das suas formas ${ }^{1-3}$. André ${ }^{1}$ propôs uma classificação das mamas em 7 tipos básicos, com subtipos A e B, exceto os tipos 2, 6 e 7, que apresentam características próprias, indicando procedimentos específicos para cada tipo de mama, sendo o tipo 6 classificada como gigantomastia.

A mamaplastia redutora é realizada para alívio dos sintomas físicos dolorosos da gigantomastia. Mulheres que sofrem de gigantomastia também referem ao cirurgião plástico grande insatisfação da imagem corporal e mudanças comportamentais em resposta ao tamanho das mamas ${ }^{4}$.
Indicações para cirurgia de redução das mamas incluem dor cervical, dor no ombro e rash cutâneo no sulco inframamário. Estudos prévios têm estabelecido que a cirurgia de redução de mamas bilateral é altamente efetiva em aliviar estes sintomas 5 .

$\mathrm{O}$ aumento das mamas durante a puberdade e no período gestacional é um processo fisiológico normal, mas um aumento exagerado e alargamento difuso durante a gestação é uma condição rara referida como gigantomastia, como sugerido por Strombeck ${ }^{6}$.

Alcançar um formato estético, estabilidade de resultados por longo tempo e menores cicatrizes é um desafio para todo o cirurgião plástico, como evidenciado pela multiplicidade de técnicas, táticas cirúrgicas e modificações que variam com atenção para a posição e tamanho das cicatrizes, escolha do pedículo de segurança e métodos de redução da mama. Técnicas comumente utilizadas previam cicatrizes no sulco mamário e periareolar ${ }^{7}$, peri-areolares, verticais e submamárias ( $\mathrm{T}$ invertido $)^{6-15}$, peri-areolares e verticais ${ }^{16-18}$, peri-areolares, verticais e submamárias laterais $(\mathrm{L})^{19,20}$, e peri-areolares isoladas ${ }^{3,21}$.

\section{MÉTODO}

Foram incluídas neste estudo 200 pacientes submetidas à mamaplastia redutora, realizadas no período compreendido

1. Membro Titular da SBCP; Cirurgião Plástico do Hospital Regional Hans Dieter Schmidt, Joinville, SC, Brasil; Membro Titular da FILACPS; Coordenador de Cirurgia Plástica do Hospital Regional Hans Dieter Schmidt, Cirurgião Plástico da OBESIMOR - Referência no tratamento da Obesidade Mórbida em Santa Catarina; Preceptor da Residência de Cirurgia Geral do Hospital Regional Hans Dieter Schmidt.

2. Médica Residente de Cirurgia Geral do Hospital Regional Hans Dieter Schmidt, Joinville, SC, Brasil. 
entre fevereiro de 1992 e setembro de 2010. Foram selecionadas para o estudo as pacientes consideradas portadoras de gigantomastia ou mama tipo 6 , conforme classificação proposta pelo autor, tendo as seguintes características: mamas com grande alargamento da base e grande projeção ântero-posterior, acompanhada de acentuada ptose e/ou flacidez cutânea, com a distância entre os pontos "A" e "M" maior que $10 \mathrm{~cm}$, sendo o ponto " $A$ " aquele que se projeta do sulco mamário à posição ideal do complexo aréolo-mamilar (CAM) (Figura 1).

\section{Procedimentos adotados}

1. Mamaplastia em "L", conforme técnica descrita por Bozzola ${ }^{2}$, com modificações pessoais do autor: compensação da pele excedente para medial, resultando em uma cicatriz final em T (Figura 2);

2. Mamaplastia em T, com enxertia do CAM, conforme técnica descrita por Marino ${ }^{15}$, com modificações pessoais do autor. As modificações por nós adotadas são: Compensações dos excessos cutâneos medial e lateral para o centro mamário, dessa forma limitando a extensão da cicatriz, entre a borda do xifóide e a linha axilar anterior; Ressecção do excedente de pele da cicatriz vertical, resultante da

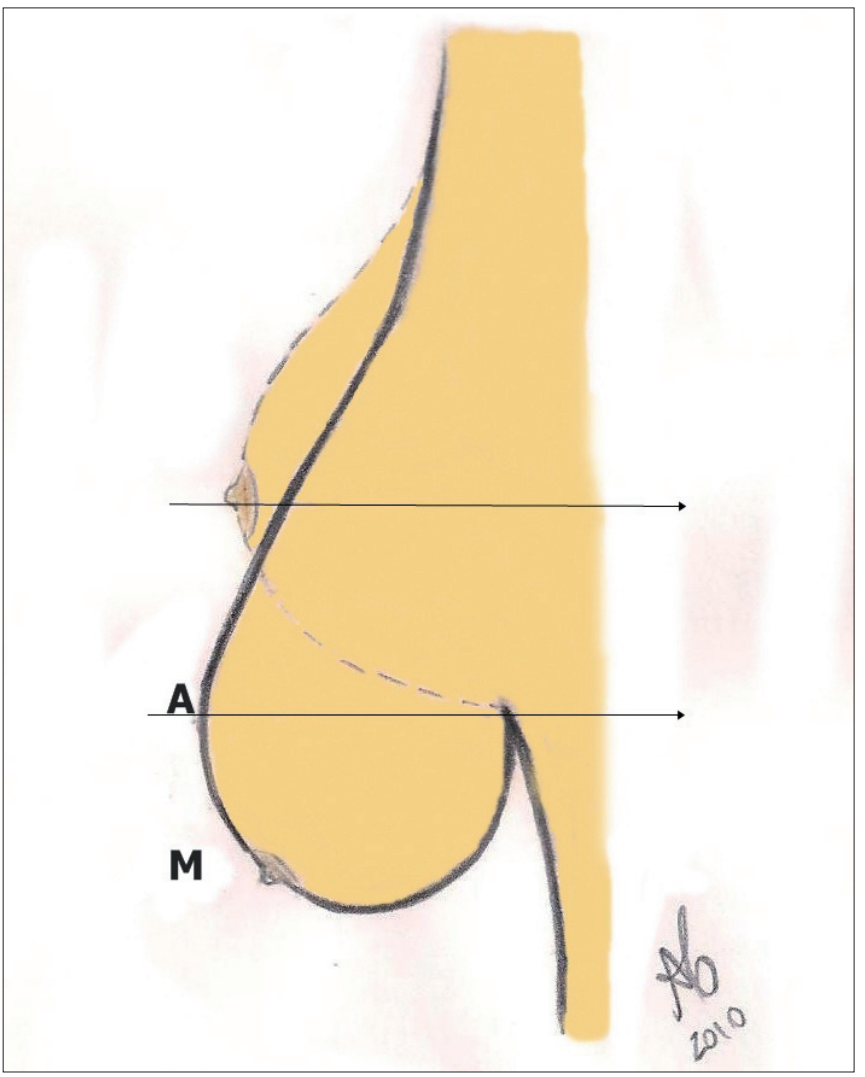

Figura 1 - Distância AM.

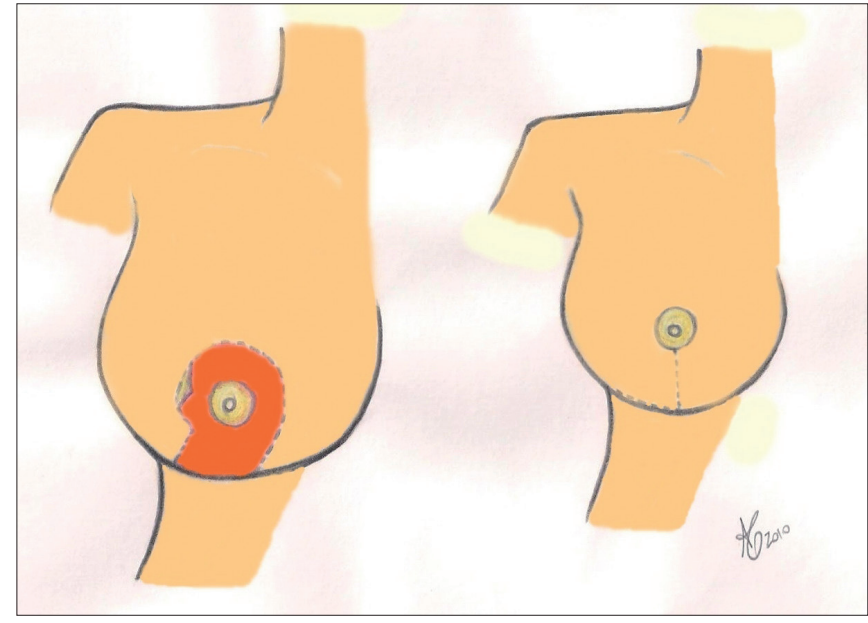

Figura 2 - Marcação em L.

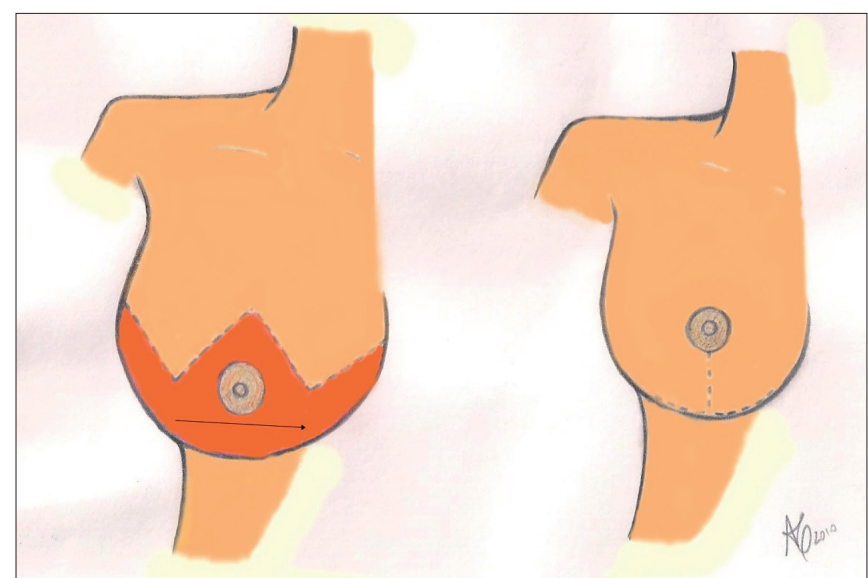

Figura 3 - Marcação em T.

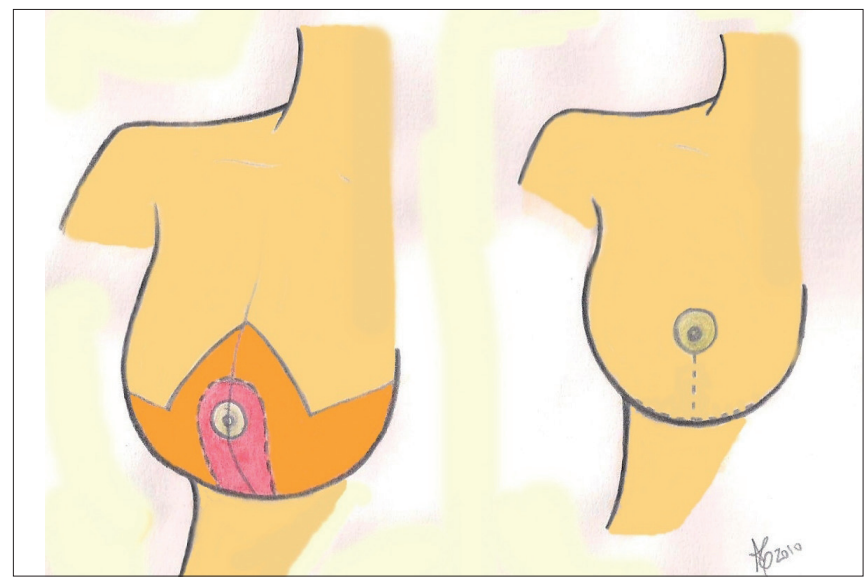

Figura 4-Marcação T, retalho inferior areolado. 
compensação cutânea; Montagem do cone mamário às custas de suturas com Mononylon 2-0 (Figura 3).

3. Mamaplastia em T, com retalho de pedículo inferior areolado com marcação descrita por Jurado e modificada pelo autor. As modificações são as mesmas adotadas para a Técnica de Marino ${ }^{15}$ (Figura 4).

\section{Preparo pré-operatório}

O preparo pré-operatório é o mesmo realizado para as demais cirurgias plásticas:

1. Avaliação clínica - diagnóstico da forma e do grau de ptose das mamas (escolha da técnica); Investigação sobre doenças pregressas das mamas, uso de drogas e medicamentos, tabagismo, gravidez.

2. Avaliação laboratorial (hemograma completo, coagulograma, TAP, glicemia, ecocardiograma, ultrassonografia de mamas e/ou mamografia).

3. Avaliação anestesiológica.

4. Orientação para cessar o tabagismo por pelo menos 4 semanas antes da cirurgia e a complicações relacionadas ao procedimento operatório.

\section{Cuidados pós-operatórios}

1. Drenagem, com drenos Penrose nas primeiras 24 horas. Eventualmente, uso de dreno tipo Suctor, nos casos de volume mamário muito grande ou acentuado sangramento intra-operatório;

2. Antibioticoterapia profilática (cefazolina $2 \mathrm{~g}$, via endovenosa, na indução anestésica e $1 \mathrm{~g}$, via endovenosa, de $6 \mathrm{em} 6$ horas, por 24/48 horas). Cefalexina $500 \mathrm{mg}$, de 6 em 6 horas, por 5 dias após a alta hospitalar;

3. Curativos com pomada antibiótica e fita microporosa, trocados no $1^{\circ}, 7^{\circ}$ e $14^{\circ}$ dia de pós-operatório e mantida por 60 dias com trocas a cada três dias;

4. Curativo de Brown nos casos de enxerto de CAM, retirado aos 5 dias de pós-operatório;

5. Uso de sutiã anatômico sem costuras por 60 dias;

6. Solicitação de exame anatomopatológico;

7. Repouso: restrição a movimentos bruscos ou intensos nos primeiros 30 dias. Liberação gradual após esse período;

8. Alta hospitalar após 24/48 horas de pós-operatório.

\section{RESULTADOS}

No período entre fevereiro de 1992 e setembro de 2010, realizamos 200 procedimentos de mamaplastia redutora para correção de gigantomastias. A idade das pacientes variou entre 16 e 73 anos.

As diferentes técnicas empregadas foram: 153 mamaplastias em "L" com modificação para o formato da cicatriz final em "T"; 40 com enxertia do CAM, e 7 mamaplastias com retalho de pedículo inferior areolado (Figuras 5 a 12).
A anestesia geral foi tipo anestésico mais utilizado, balanceada ou endovenosa total (150 pacientes), peridural (46 pacientes) e peridural + endovenosa total (4 pacientes - falha técnica).

O volume mamário ressecado variou entre $650 \mathrm{~g}$ e 3555 g por mama (Figura 13).

O tempo de internação foi de até 2 dias de pós-operatório para todas as pacientes.

Observamos um caso de hematoma, um caso de seroma e nenhuma infecção da ferida operatória. Relatamos 9 casos de perda total do enxerto areolar (unilateral), 10 casos de perda parcial do enxerto do CAM (unilateral) e 15 casos com deiscências parciais de sutura, na junção das cicatrizes vertical e horizontal.

Observamos que 188 pacientes apresentaram cicatrizes eutróficas no pós-operatório, sendo as 200 pacientes avaliadas por pelo menos 180 dias após a cirurgia. Observamos, também, 3 pacientes com cicatriz hipertrófica, 7 com cicatrização por segunda intenção e 2 pacientes com cicatrizes quelóides.

As formas das mamas apresentaram-se todas relativamente regulares após a cirurgia, mas notamos a irregularidade das aréolas em 15 pacientes. Todas as pacientes referiram melhora dos sintomas que indicavam a cirurgia: cervicalgia, dorsalgia, dor no ombro, dermatite inframamária, insônia, etc.

$\mathrm{Na}$ análise anatomopatológica, encontramos uma amostra com papiloma intraductal e, nas demais, os achados eram normais ou alterações benignas, como hiperplasia acinar e ductal, congestão venosa, alterações fibrocísticas da mama, ectasia ductal, fibrose com hialinização, metaplasia apócrina, adenose esclerosante.

\section{DISCUSSÃO}

Inúmeras técnicas e táticas cirúrgicas foram descritas ao longo de 8 décadas, com uma grande variedade de abordagens. Os resultados obtidos nem sempre são semelhantes.

Adotamos 3 procedimentos: a mamaplastia em $\mathrm{T}$, com enxertia do CAM, baseada na técnica descrita por Marino ${ }^{15}$, com modificações pessoais. Mamaplastia com marcação em $\mathrm{L}\left(\right.$ Bozzola $\left.^{2}\right)$ que transformamos em uma cicatriz final em T.

Mamaplastia em T, com técnica modificada de Marino ${ }^{15}$, como descrito anteriormente e utilizando retalho de pedículo inferior areolado (Jurado).

Adotamos uma rotina de classificação e indicação da técnica, com o objetivo de obter maior previsão dos resultados cirúrgicos. Outras técnicas podem ser utilizadas, mas com essas descritas é que obtivemos nossos melhores resultados.

Pacientes que se submetem à redução de mama apresentam melhora importante da relação saúde-qualidade de vida. A melhora é notada entre o $1^{\circ}$ dia antes da cirurgia e 1 

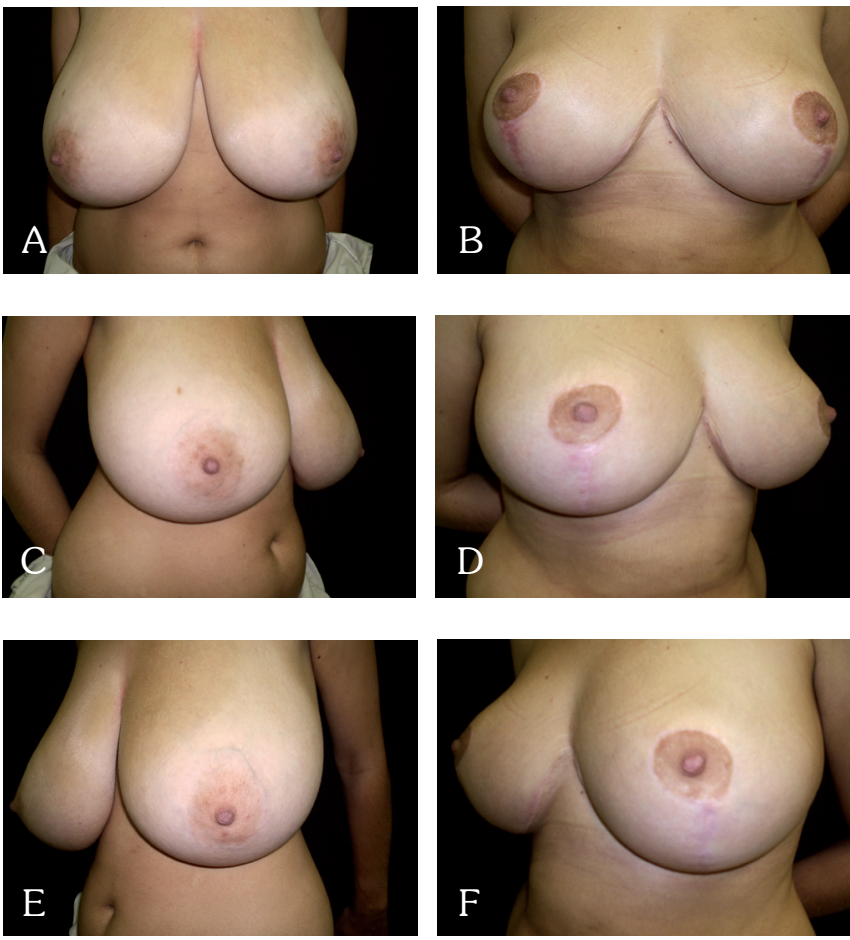

Figura 5-A: Mamaplastia LT pré-AP. B: Mamaplastia LT pós AP. C: Mamaplastia LT pré OBD. D: Mamaplastia LT pós OBD. E: Mamaplastia LT pré OBE. F: Mamaplastia LT pós OBE.
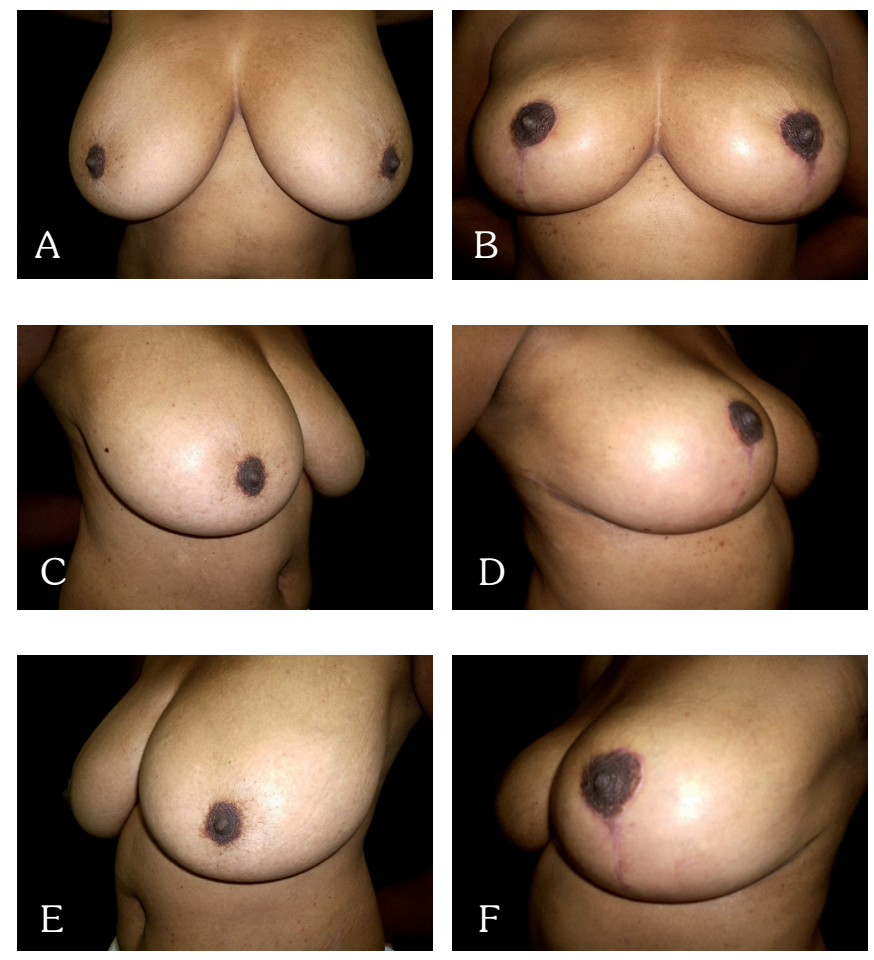

Figura 6-A: Mamaplastia LT pré AP. B: Mamaplastia LT pós AP. C: Mamaplastia LT pré OBD. D: Mamaplastia LT pós OBD. E: Mamaplastia LT pré OBE. F: Mamaplastia LT pós OBE.
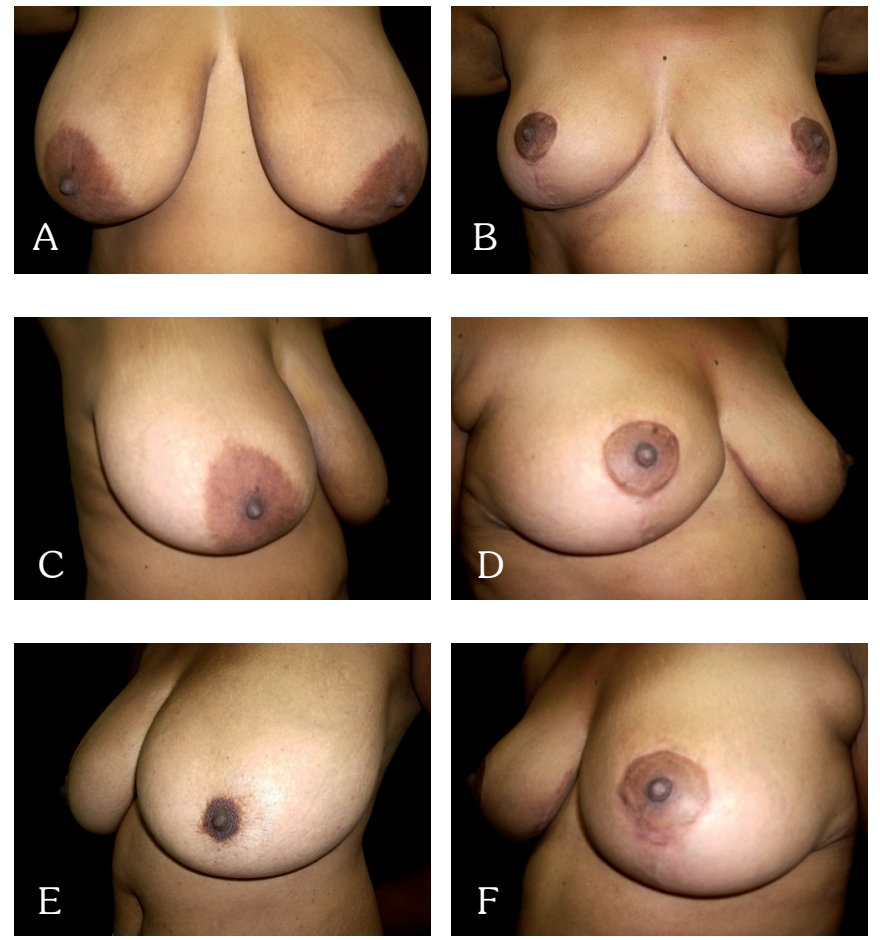

Figura 7-A: Mamaplastia LT pré AP. B: Mamaplastia LT pós AP. C: Mamaplastia LT pré OBD. D: Mamaplastia LT pós OBD. E: Mamaplastia LT pré OBE. F: Mamaplastia LT pós OBE.
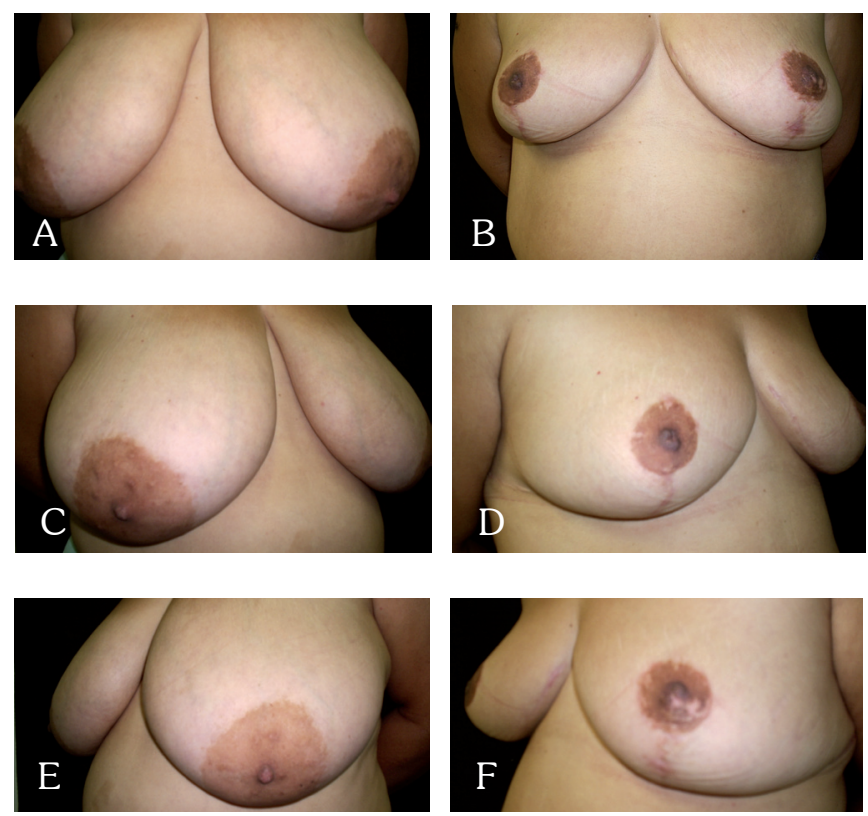

Figura 8-A: Mamaplastia T com retalho de pediculo inferior areolado pré AP. B: Mamaplastia com retalho de pedículo inferior areolado pós AP. C: Mamaplastia com retalho de pedículo inferior areolado pré $O B D$. D: Mamaplastia com retalho de pediculo inferior areolado pós $O B D$. E: Mamaplastia com retalho de pedículo inferior areolado pré OBE. F: Mamaplastia com retalho de pediculo inferior areolado pós $O B E$. 

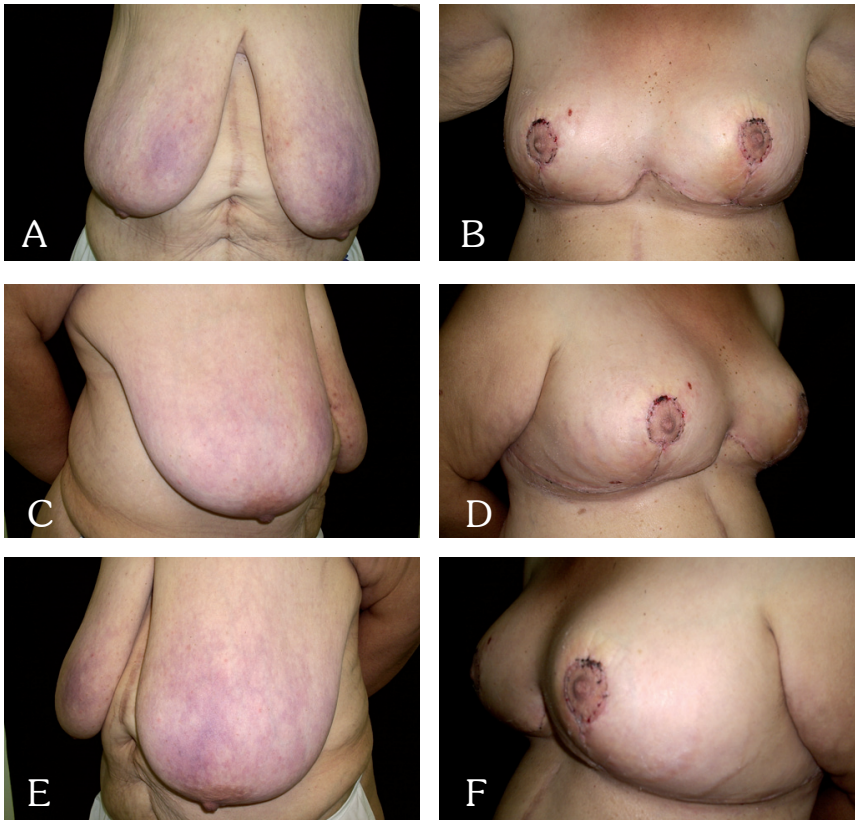

Figura 9-A: Mamaplastia com retalho de pediculo inferior areolado pré AP. B: Mamaplastia com retalho de pediculo inferior areolado pós AP. C: Mamaplastia com retalho de pediculo inferior

areolado pré $O B D$. D: Mamaplastia com retalho de pediculo

inferior areolado pós $O B D$. E: Mamaplastia com retalho de pediculo inferior areolado pré $O B E$. F: Mamaplastia com retalho de pedículo inferior areolado pós $O B E$.
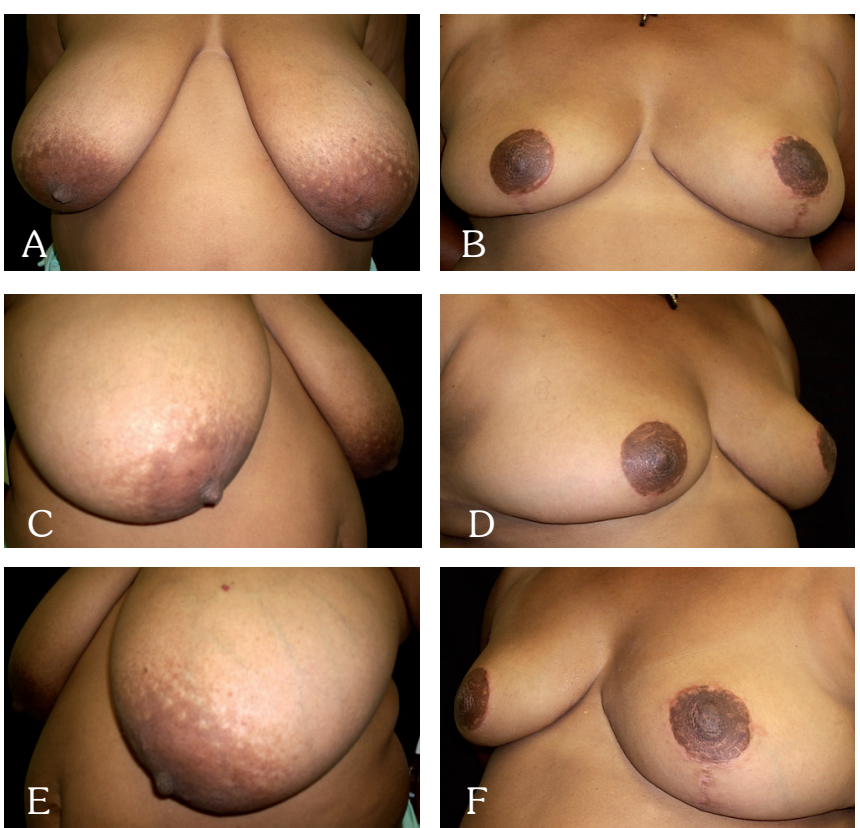

Figura 10 - A: Mamaplastia em T com enxertia do CAM pré AP. B: Mamaplastia em com enxertia do CAM pós AP. C: Mamaplastia em $T$ com enxertia do CAM pré OBD. D: Mamaplastia em T com enxertia do CAM pós OBD. E: Mamaplastia em T com enxertia do CAM pré

OBE. F: Mamaplastia em T com enxertia do CAM pós OBE.
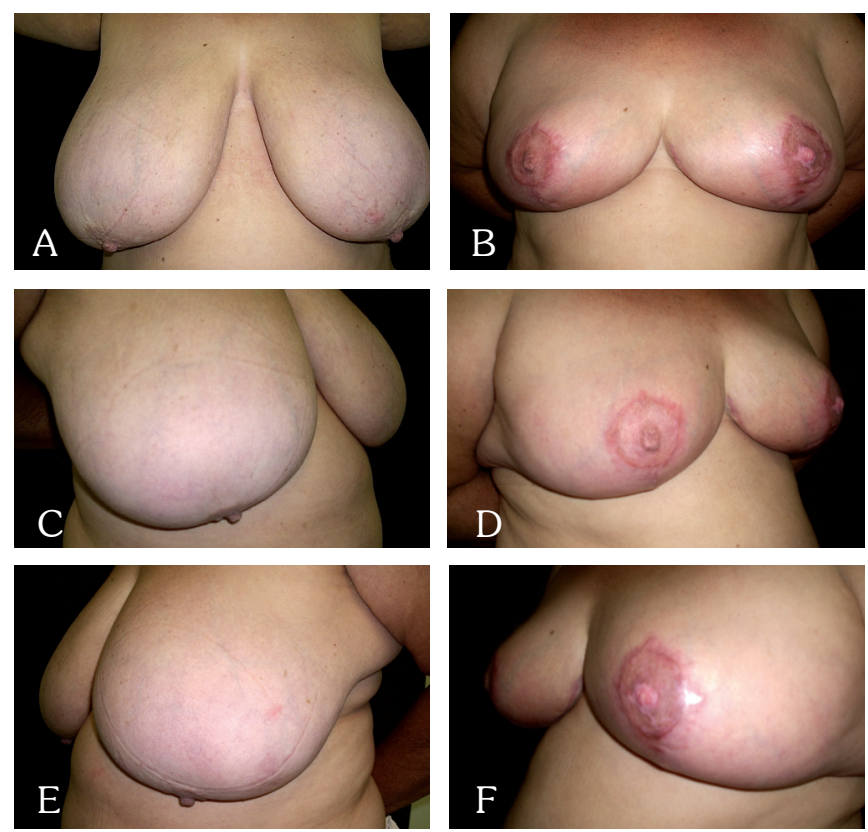

Figura 11 -A: Mamaplastia em T com enxertia do CAM pré AP. B: Mamaplastia em T com enxertia do CAM pós AP. C: Mamaplastia em $T$ com enxertia do CAM pré OBD. D: Mamaplastia em T com enxertia do CAM pós OBD. E: Mamaplastia em T com enxertia do CAM pré

$O B E$. F: Mamaplastia em T com enxertia do CAM pós $O B E$

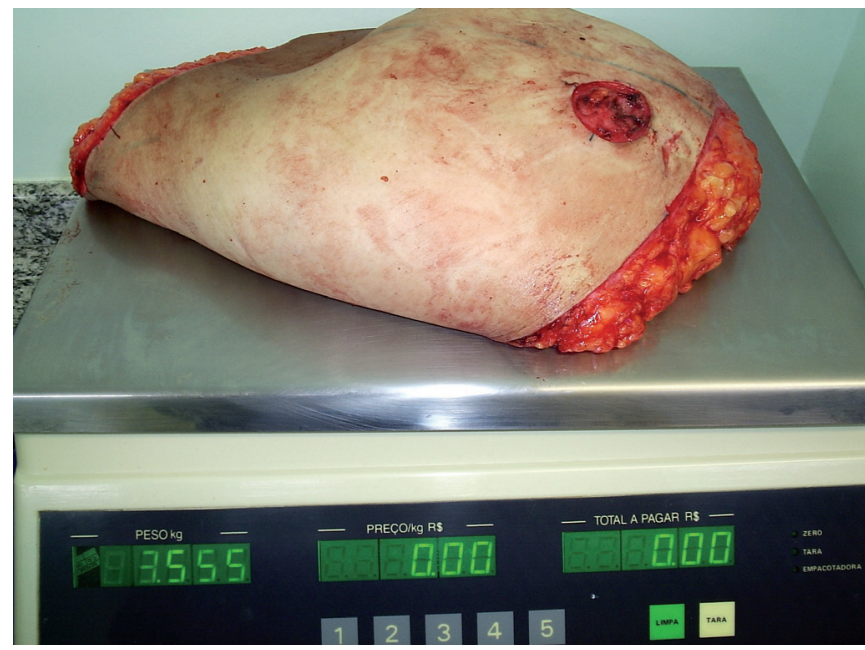

Figura 12 - Maior volume mamário ressecado.

mês após, depois disso estabiliza por até 1 ano. A redução de mama melhora significativamente qualidade de vida, a sociabilidade e a estabilidade emocional.

Em casos de gigantomastia importante, o enxerto do CAM tem sido a alternativa tradicional à transposição de pedículo. Um estudo com 142 pacientes demonstrou que não houve diferença estatística quanto à sobrevivência da aréola 
ou outras complicações em pacientes submetidas a grandes reduções ( $>1500 \mathrm{~g}$ por mama) comparado a pequenas reduções $(<1500 \mathrm{~g}$ por mama $)$.

\section{CONCLUSÃO}

Após análise dos dados coletados, concluímos que o objetivo principal da realização da mamaplastia redutora foi alcançado em todas as pacientes. Tínhamos como objetivo principal para a realização da cirurgia de redução de mama a alívio dos sintomas relacionados ao aumento excessivo da mama, como dores crônicas dorsal, cervical, no ombro, dermatite inframamária, insônia, além da melhora na qualidade de vida. A escolha da técnica/tática cirúrgica ideal para cada caso foi fundamental para obtermos os resultados obtidos.

\section{REFERÊNCIAS}

1. André FS. Mammary arts planning: new classification of mammary shapes. Arq Catarin Med. 2007;36(Supl. I):55-8.

2. Bozzola A. Mamaplastia em "L". In: Ribeiro L, ed. Cirurgia plástica da mama. Rio de Janeiro:Medsi;1989. p.7129-83.

3. Rees TD. An hystorical review of mammaplasty. Anais do Transact Fifht Intern Cong Plast S; Austrália.

4. Glatt BS, Sarwer DB, O'Hara DE, Hamori C, Bucky LP, LaRossa D. A retrospective study of changes in physical symptoms and body image after reduction mammaplasty. Plast Reconstr Surg. 1999;103(1):76-82.

5. Strombeck JO. Macromastia in women and its surgical treatment. A clinical study based on 1,042 cases. Acta Chir Scand Suppl. 1964;341(Su- ppl):1.

6. Strombeck JO, Mammaplasty in hypertrophy of the female breast. In: III Congress Transaction Intern Soc Plast Surg; 1967; Washington, USA.

8. Thorek M. Plastic reconstruction of the breast and free transplantation of the nipple. J Int Coll Surg. 1946;9:124-24.

9. Lexer E. Die gesante wederherstelung chirurgie. Leipzig J A Brath. 1931.

10. McKissock PK. Reduction mammaplasty with a vertical dermal flap. Plast Reconstr Surg. 1972;49(3):245-52.

11. Peixoto G. Reduction mammaplasty: a personal technique. Plast Reconstr Surg. 1980;65(2):217-26.

12. Pitanguy I. Breast hypertrophy. Anais do II Congress of International Society of Plast Surgeons; 1959; Londres, Inglaterra.

13. Ribeiro L, Backer E. Mastoplastia com pediculo de seguridad. Rev Esp Cirurg Plast. 1973;6:223.

14. Skoog T. A technique of breast reduction: transposition of the nipple on a cutaneous vascular pedicle. Acta Chir Scand. 1963;126:453-65.

15. Marino H. La mama. Buenos Aires:Editorial Cientifica Argentina;1957.

16. Ariê G. Nova técnica em mamaplastia. Rev Lat Amer Cir Plast. 1957;3:28.

17. Ely JF. The devil's incision mammoplasty. Aesthetic Plast Surg. 1983;7(3):159-62.

18. Lejours H. Vertical mammaplasty an liposuction of the breast. St Louis:Quality Medical Publishing;1993.

19. Chiari Jr A. The L short scar mammaplasty: a new approach. Plast Reconst Surg. 1992; 90:233.

20. Andrews JM, Yshizuki MM, Martins DM, Ramos RR. An areolar approach to reduction mammaplasty. Br J Plast Surg. 1975;28(3):166-70.

21. Bustos RA. Periareolar mammaplasty with silicone supporting lamina. Plast Reconst Surg. 1992;89(4):646-57.

\section{Correspondência para:}

Fernando Sanfelice André

Rua Osvaldo Cruz, 128 - Bairro Boa Vista - Joinville, SC, Brasil - CEP 89205-240

E-mail: sanfi@brturbo.com.br 